\title{
Clinical Efficacy of a New Filmogen Polymeric Glycerol Solution for the Treatment of Rhinosinusitis
}

\author{
Shrivastava RM ${ }^{1^{*}}$, Tourret $E^{1}$, Schutte $\mathrm{H}^{2}$, Shrivastava $\mathrm{RS}^{1}$ and Roura $\mathrm{F}^{3}$ \\ ${ }^{1}$ Vitro-Bio Research Institute, Issoire, France \\ ${ }^{2}$ Strathos Pharma Group, Bad Ems, Germany \\ ${ }^{3}$ REVA Health S.L, 08195 Barcelona, Spain
}

*Corresponding author: Shrivastava RM, Vitro-Bio Research Institute, ZAC de Lavaur, Issoire, France, Tel: +330473550505; Fax: +330473550011; E-mail: rs@vitrobio.com

Received date: June 3, 2017; Accepted date: June 25, 2017; Published date: July 10, 2017

Copyright: (c) 2017 Shrivastava RM. This is an open-access article distributed under the terms of the Creative Commons Attribution License, which permits unrestricted use, distribution, and reproduction in any medium, provided the original author and source are credited.

\begin{abstract}
Study background: The main cause of chronic rhinosinusitis is not yet well understood but increasing amount of evidence suggest an initial viral attack followed by secondary bacterial infection leading to the formation of a wellprotected reservoir of microorganisms in the sinuses where no treatment can easily reach. The efficacy of a new, non-irritant and moderately osmotic, polymeric glycerol filmogen solution was evaluated clinically.
\end{abstract}

Methods: A 14-day, randomized, placebo-controlled, double blind, efficacy and safety clinical study was conducted with participation of thirty-eight patients treated with the filmogen polymeric glycerol vs. saline solution as comparator treatment used on sixteen control patients. Two to three nasal sprays were applied, three times daily over maximum fourteen days. Effects on rhinorrhea, nasal congestion, headache, facial pain, and sino-nasal outcome test were evaluated.

Results: Rhinorrhea was much stronger in the test product group during the 1st three days compared to comparator and decreased drastically thereafter. Severity of all other rhinosinusitis symptoms also diminished in both groups but the reduction was much faster and stronger in the test group compared to comparator group $(p<0.05)$, and led to minimized requirement for antibiotics. The test product was non-irritant and totally safe, no adverse effects being observed.

Conclusion: Treating rhinosinusitis with a moderately osmotic solution capable of generating positive osmotic pressure yet non-irritant polymeric film constitutes a major breakthrough in the treatment of Rhinosinusitis.

Keywords: Filmogen polymeric glycerol; Clinical; Rhinosinusitis; Sinus; Positive osmotic pressure

\section{Introduction}

Rhinosinusitis (RS) or Chronic rhinosinusitis (CRS) usually starts by a viral infection, leading to the destruction of nasal mucosa cells, inflammation and edema of the middle meatus and/or ethmoidal part and nasal mucosa, followed by secondary bacterial infection and purulent nasal discharge or nasal obstruction [1].

Up to this stage, the disease can be treated easily but if the infection is not stopped microorganisms enter into the nasal sinuses and start multiplying in this highly favorable and protected environment. The microorganisms particularly bacteria and fungi act symbiotically to protect themselves against all aggressions: the sessile planktonic bacteria adhere to the sinus surface, secrete protective extracellular matrix and form 3-dimensional biofilm aggregates of microorganisms $[2,3]$.

The number of these biofilm aggregates increase with time, damage sinus mucosa and may obstruct sinus clearance. Biofilms harbor 10-1000-fold higher resistance to antimicrobials than planktonic bacteria due to a strong physical barrier to all external attacks. Chronic sinus infection leads to increased intra-sinus pressure, facial pain and headache and occasionally development of polyps in the nasal cavity. Rhinosinusitis is defined as chronic when it lasts over 12 weeks without complete symptom resolution [4]. According to a US national health interview survey, CRS has been estimated to affect $12.5 \%$ to $15.5 \%$ of the total population, making it the second most prevalent chronic condition in the United States [5].

However, according to the international statistical classification of diseases and related health problems, hardly $2 \%$ rhinosinusitis patients visit doctors [6]. This is particularly due to the lack of effective treatments because the nasal mucosa is one of the most sensitive organs in the body and becomes easily irritated or damaged whereas sinuses are empty poorly irrigated cavities that drugs cannot easily reach and there is no device to effectively eliminate bacterial biofilm $[2,6]$.

Current treatments of CRS include single target oriented medical or surgical therapy. CRS being a multi-factorial disease its medical therapy often requires combining multiple medications including antibiotics, topical nasal steroids and/or oral steroids, and saline irrigation.

These treatments are aimed at providing symptomatic relief, reducing the bacterial load, facilitating nasal drainage, minimizing secondary infection or reducing edema or inflammation but many 
patients do not respond to these treatments satisfactorily because they act on a specific target and have no effect on biofilm. Therefore, to be effective the basic treatment strategy should be directed at disrupting biofilms to relieve intra-sinus infection and pressure, normalize sinus drainage and provide permanent relief [7].

In the absence of any other safe treatment, saline solution containing $0.9 \%$ to $3.2 \% \mathrm{NaCl}$ still constitutes a treatment of choice as it helps to clean the nasal passage, ease respiration and improve ciliary movements but they have no effect on the basic cause of the disease [8]. We conceived a moderately hypertonic, long-acting polymeric film capable of generating positive osmotic pressure over the nasal mucosa to treat RS.

It was postulated that such a film should protect the nasal mucosa against irritation, should attract some hypotonic liquid to clean the nasal surface and should probably improve sinus drainage. The clinical efficacy of this filmogen polymeric glycerol (FPG) solution $[9,10]$ was evaluated in comparison against saline solution.

\section{Materials and Methods}

\section{The test product (TP)}

The TP contained a diluted glycerol-based solution (VB-Gy) which is nearly 18 times more osmotically active than 3.2 to $3.4 \% \mathrm{NaCl}$ sea water yet not as irritant [9]. Glycerol is a natural antiseptic and cellfriendly solution. Its properties make glycerol an ideal candidate which can exert sufficient osmotic pressure over semi-permeable live biological membranes without being irritable to the nasal mucosa or being cytotoxic to the already damaged cells.

We postulated that the topical application of such a solution should attract hypotonic liquid from the semipermeable membrane and if the pressure exerted is sufficiently strong it may clean the surface of contaminants and improve sinus drainage. As VB-Gy alone would get diluted with the strong hypotonic liquid outflow it generates; it was improved to become filmogen, flexible and more resistant to dilution by adding a small quantity of Rhinocyanidins. Rhinocyanidins contain a specific association of natural polymeric ingredients such as plant extracts, natural gums (Solagum), plant cellulose (Hydroxypropylmethyl cellulose) and hydrophobic essential oils capable of enhancing VB-Gy filmogenicity and resistance to hypotonic liquid flow.

The resulting filmogen solution is termed Filmogen Polymeric Glycerol (FPG), different compositions of which are patented [10] Pharmacological studies conducted in our laboratory (unpublished confidential data) show that even low concentration (5-10\%) of FPG form highly water-resistant and flexible films having strong osmotic properties. Saline solution $(0.9 \% \mathrm{NaCl})$ was used as a comparator product $(\mathrm{CP})$.

\section{Clinical study organizer}

The clinical part of this research was conducted at Nexus Clinical Research Center in India affiliated to Nexus Clinical Research LLC, USA. The protocol and study design were approved by the Institutional Ethical Committee of India-Rajiv Gandhi Institute of medical sciences (EC Registration $N^{\circ}$ ECR/492/Inst/AP/2013, dated 05/12/2013) and the trial was conducted following the ICH-GCP guidelines as per the declaration of Helsinki concerning ethical principles for medical research involving human subjects.

\section{Study design and rationales}

The study was designed as a multi-centric, randomized, placebocontrolled, double-blind, clinical trial. The aim of the study was to compare the efficacy and safety of FPG osmotic filmogen nasal spray with a commonly used treatment: physiological saline solution as comparator for the treatment of CRS. The doses were selected based on dose range findings from a previous pilot observational study with CRS patients where FPG treatment was administered 4 times per day for 14 days without any side effects.

$0.9 \% \mathrm{NaCl}$ saline spray was chosen as comparator product $(\mathrm{CP})$ as saline solution is commonly used for symptomatic relief of rhinosinusitis and it is applied identically to the TP. The number of patients enrolled was defined based on the minimum number of patients required in such type of studies to obtain statistically comparable data between two groups. The efficacy of saline solution in CRS being well known, it was decided to enroll more patients in the TP group. The requirement for the use of antibiotics was also assessed as antibiotherapy is very often employed for CRS. It's worth mentioning that bacterial resistance is becoming a serious health concern for CRS patients due to repeated long-term treatments.

\section{Inclusion and exclusion criteria}

At the time of recruitment at the study centers, patients were examined physically and patient's medical, surgical, and allergic history was checked and recorded. Vital signs such as blood pressure, pulse rate, and respiratory rate were recorded. Patients not suffering from any serious pathology were then examined for enrollment in the study.

The main inclusion criteria comprised: informed consent (signed form) for participation in the study males or females aged 18-70 years; not under any treatment which may affect study outcome (CRS treatments, antibacterial, antiviral treatment, anti-histamines or steroids); no nasal surgery in past year; having clinical manifestations of CRS with a minimum rhinosinusitis severity score (RSS) of 10 out of 20. RSS was calculated as sum of the scores assessing the severity on a 0 (not present) to 4 (very severe) scales, of the following symptoms:

- Purulent nasal discharge

- Nasal obstruction or congestion

- Facial pain

- Headache and swelling around the eyes

- Sore throat or pharyngitis.

The main exclusion criteria included:

- Presence of any other respiratory disease

- Being under medication

- Known allergy to test product (TP) components

- Lack of willingness to participate in the study

\section{Randomization}

After screening, patients satisfying all the inclusion criteria were enrolled and randomly allocated in a 2:1 ratio to either group:

- TP group patients received the hypertonic filmogen test product and

- $\mathrm{CP}$ group patients received the $0.9 \% \mathrm{NaCl}$ solution as comparator product 
Page 3 of 8

Treatments were allocated to patient by carrying out randomization using SAS Version 9.1.3 following a randomization schedule. Block Randomization methodology was employed for generating the list. Within the block the treatments were distributed in the ratio of $2: 1$ as mentioned above. Each patient received a unique screening identification number, randomization code, and enrollment identification number.

\section{Product presentation and administration}

TPs and CPs were supplied by NATURVEDA SAS, France (ISO 13485 certified) and were presented identically ( $15 \mathrm{ml}$ spray containing slightly viscous and colorless liquid) except for the product code and the batch number.

The TPs contained an aqueous solution of FPG with Rhinocyanidins while $\mathrm{CPs}$ contained $0.9 \% \mathrm{NaCl}$ saline solution. Patients were asked to apply 2-3 pulverizations of the allocated treatment in each nostril, 3 to 4 times per day (morning, mid-day, evening and before night rest) from Day 1 to Day 14 or till complete recovery whichever was earlier. $1^{\text {st }}$ treatment was administered just after the patient's inclusion in the study (Day 1).

\section{Parameters studied}

The primary outcome was defined as changes in rhinosinusitis symptom severity which was evaluated on a rating scale of 0 to $10(0$ meaning absence of symptoms) just before $1^{\text {st }}$ treatment and on days 1 , 3,6 and 14 by the patients and the symptom severity scores were recorded in the patient diary. Key parameters included rhinorrhea, nasal congestion, headache, facial pain upon pressure and change in the mean values of overall major symptom scores.

The 22 parameters as per Sino-Nasal Outcome Test (SNOT-22) described by Brown et al. were also measured as secondary endpoint at the start and at the end of the study on a 0 (no problem) to 5 (severe problem) severity scale [11]. Total score and mean values of SNOT-22 parameters were evaluated in TP compared to CP.

The main secondary outcome criteria also included the necessity of employing antibiotics and product acceptability. Screening values were considered as baseline values and for ethical reasons treatment was started just after patient's enrollment in the study (Day 1). Safety assessment: at the end of the study subjects and investigators evaluated any eventual treatment-emergent adverse effects as well as tolerability and acceptability of TP and CP.

\section{Statistical analysis of results}

The analyses were conducted with Microsoft Excel and XLStat using the available data. Significant effects were those with a probability lower than $\alpha=0.05$. Statistically significant results were indicated in abbreviated manner: SS and not statistically significant results ( $p>0.05)$ : NS.

For each score repeated measures analysis of variance (RMANOVA) determined differences in symptoms scores across study visits. Nemenyi post-hoc test provided pairwise comparisons in a group between baseline and the end of the study. Results were compared with baseline values (before $1^{\text {st }}$ treatment on Day 1 ) in the same group and between the groups at each time point.

\section{Results}

\section{Demographics}

Study population that completed the study consisted in 38 patients (26 $\mathrm{M}$ and $12 \mathrm{~F}$ ) aged $21-55$ years (mean 35.86 years) in the TP group and 16 patients ( $13 \mathrm{M}$ and $3 \mathrm{~F}$ ) aged $23-49$ years (mean 34.25 years) in the $\mathrm{CP}$ group. The distribution of patients based on physical assessments was homogenous between the groups.

At the time of recruitment, all enrolled subjects were diagnosed with moderate to severe clinical manifestations of Rhino-sinusitis (RS) including major symptoms (rhinorrhea (anterior nasal discharge, postnasal drip, nasal congestion, headache and facial pain/pressure), with a minimum Rhino-sinusitis severity (RSS) score of 25 out of 50 (Figure 1).

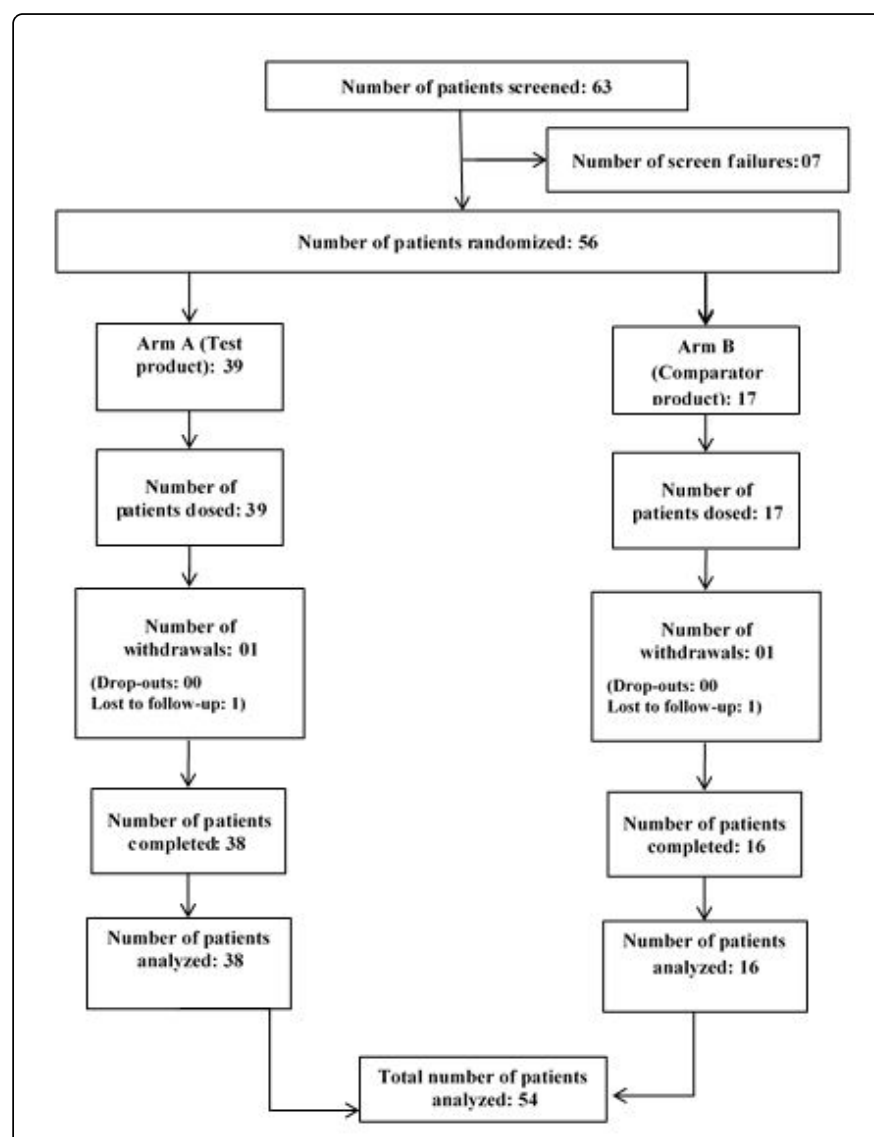

Figure 1: Population distribution flowchart.

Patients with diagnosis of respiratory diseases that could compromise breathing, example: bronchopneumonia, asthma, rhinosinusitis of known fungal or allergic origin, abnormal structural narrowing of the sinus passages such as a deviated septum or any other kind of anatomical obstruction or CRS with nasal polyps, clinical evidence of immunosuppression, psychiatric diseases or major depression and patients who had recent nasal surgery were already under treatment for respiratory diseases, chronic allergy or antibacterial or antiviral treatment, anti-histamine, steroids in the 2 week prior to screening were not enrolled in the study. The baseline 
Citation: Shrivastava RM, Tourret E, Schutte H, Shrivastava RS and Roura F (2017) Clinical Efficacy of a New Filmogen Polymeric Glycerol

Page 4 of 8

mean symptom scores were fairly identical in both groups with mean severity often slightly higher in the TP group at study outset.

\section{Effect on rhinorrhea}

At the start of the study baseline rhinorrhea severity scores were relatively high in both groups with slightly more elevated mean value in the TP group (7.13/10) compared to the CP group (6.56/10) (Figure 2).

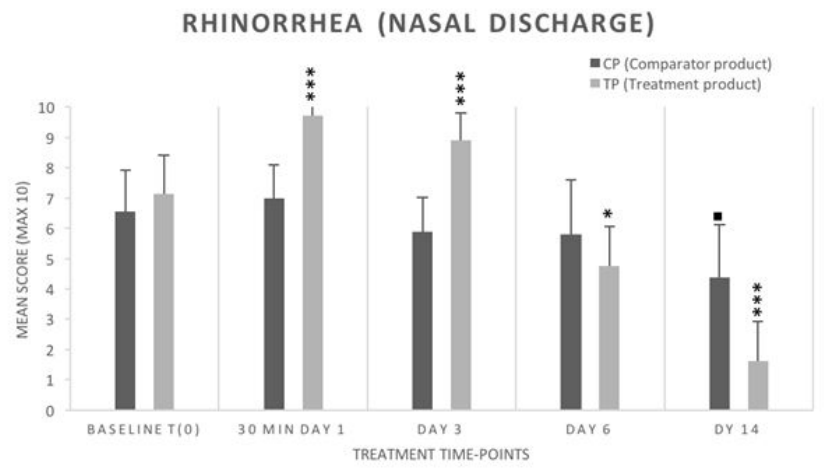

Figure 2: Mean score $( \pm \mathrm{SD})$ for rhinorrhea in comparator product group (black) vs. test product group (light gray) just before treatment (baseline T0) and on day 1 (30 minutes after $1^{\text {st }}$ application) 3, 6 and $14 .{ }^{*} \mathrm{p}<0.05,{ }^{* *} \mathrm{p}<0.01,{ }^{* *} \mathrm{p}<0.001$ for TP compared to $\mathrm{CP}$ at the same time point and $=<0.05$ compared to baseline values.

Results show that 30 minutes after the first treatment application rhinorrhea was not reduced but strongly increased in the TP group in almost all the patients with individual scores usually between $9 / 10$ or 10/10 (mean 9.71 in TP i.e. SS $36.16 \%$ increase, $\mathrm{p}<0.0001$, vs. $7.0 / 10$ in CP i.e. NS $6.66 \%$ increase, $\mathrm{p}=0.734$ ) indicating that the TP strongly and significantly increases nasal secretions after application.

Consequential nasal outflow was observed in almost all TP group patients up to Day 3 with mean score $51.96 \%$ higher (SS, $\mathrm{p}<0.0001$ ) $(8.92 / 10)$ than in the CP group (5.87/10) where nasal flow had slightly decreased on Day 3. Remarkably, rhinorrhea started abating strongly in the TP group from Day 3 onwards.

Mean scores for rhinorrhea on Days 6 and 14 were 4.76 and 1.63 in the TP group compared to 5.81 and 4.37 in the $\mathrm{CP}$ group showing a significantly( $\mathrm{p}=0.02$ Day 6 and $\mathrm{p}<0.0001$ Day 14$)$ stronger reduction of the symptom in the TP group than observed in the CP group. These findings also show that $\mathrm{CP}$ possesses only moderate anti-rhinorrhea properties as the initial score of $6.56 / 10$ was slightly increased after 30 minutes $(\mathrm{NSp}=0.73)$ followed by a reduction of $10.5 \%$ on Day 6 (NS, $\mathrm{p}=0.48$ ) and $30.7 \%$ on Day 14 (SS, $\mathrm{p}=0.008$ ) when administered regularly (3-4 times a day) for a period of 14 consecutive days.

\section{Effect on nasal congestion}

Chronic rhino-sinusitis involves nasal congestion concomitantly with rhinorrhea. The initial scores for nasal congestion were high in both TP (mean 7.28/10) and CP (6.81/10) groups. Compared to baseline values nasal congestion in the $\mathrm{CP}$ diminished slightly and progressively throughout the 14-day treatment period with a reduction of $15.6 \%, 17.4 \%, 25.7 \%$ and $39.5 \%$ after 30 minutes $(\mathrm{p}=0.06)$ and on Day $3(\mathrm{p}=0.05)$, Day $6(\mathrm{p}<0.05)$ and Day $14(\mathrm{p}<0.001)$ respectively (Figure 3).

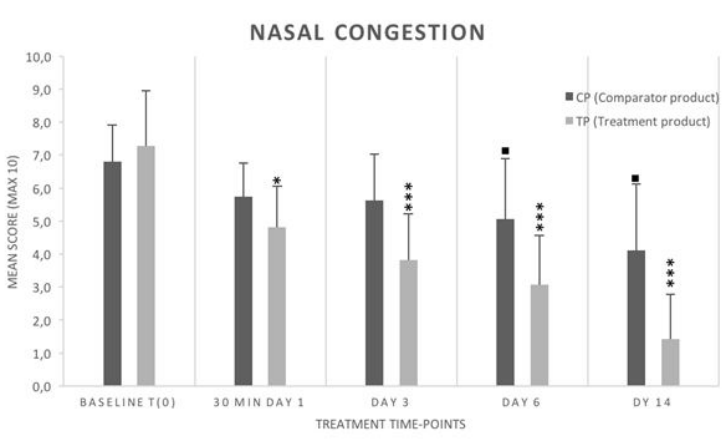

Figure 3: Mean score $( \pm \mathrm{SD})$ for nasal congestion in comparator product group (black) vs. test product group (light gray) just before treatment (baseline T0) and on days 1 (30 minutes after 1st application) 3, 6 and $14 .{ }^{*} \mathrm{p}<0.05,{ }^{* *} \mathrm{p}<0.01,{ }^{* *} \mathrm{p}<0.001$ for TP compared to $\mathrm{CP}$ at the same time point and $-<0.05$ compared to baseline values.

In the TP group nasal congestion severity scores dropped drastically in $32 / 37$ patients with significant severity decrease of nearly $34 \%$ $(\mathrm{p}=0.049)$ within 30 minutes of first treatment administration. It continued decreasing steadily and significantly $(\mathrm{p}<0.0001$ at all timepoints) afterwards by $47.6 \%, 57.8 \%$ and $80.5 \%$ on Days 3,6 and 14 respectively compared to baseline mean value and with statistically significant evolution difference from Day 3 ( $p<0.0001)$ compared to $C P$ group.

\section{Effect on headache}

Headache during rhino-sinusitis is often a result of blocked sinuses and high intra-sinus pressure. Therefore, reduction in headache is closely proportional to sinus drainage. Results show that CP had no significant $(p=0.213)$ effect on headache during the $1^{\text {st }}$ week of treatment: $16.7 \%$ reduction on Day 6 (mean score: 4.69) from T0 (mean score: 5.63) (Figure 4).

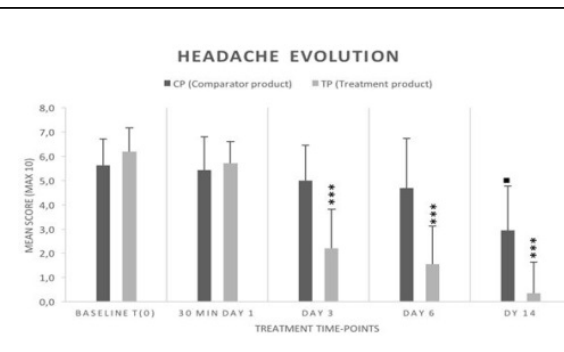

Figure 4: Mean score $( \pm$ SD) for headache in Comparator Product group (black) vs. Test Product group (light gray) just before treatment (baseline T0) and on Days 1 (30 minutes after 1st application) 3, 6 and $14 .{ }^{*} \mathrm{p}<0.05,{ }^{* *} \mathrm{p}<0.01,{ }^{* *} \mathrm{p}<0.001$ for $\mathrm{TP}$ compared to $\mathrm{CP}$ at the same time point and $-<0.05$ compared to baseline values. 
Page 5 of 8

Significant yet moderate improvement was observed by the end of the second week of treatment: on Day 14 the mean score had decreased to 2.94 indicating that saline solution does help reduce headache but requires more than 1 week of regular nasal washes to produce any significant effect.

In the TP group all patients except one showed dramatic and significant reduction of headache during the $1^{\text {st }}$ week of treatment $(\mathrm{p}<0.0001)$ and nearly no headache by Day $14(\mathrm{p}<0.0001)$. Mean scores indicate no significant effect after 30 minutes $(7.6 \%, \mathrm{p}=0.69)$ but strong and significant $(\mathrm{p}<0.0001)$ reduction of $64 \%, 75 \%$ and $94 \%$ compared to T0 values on Days 3, 7 and 14 respectively. Symptom scores showed statistically significant evolution difference between the groups from Day $3(\mathrm{p}<0.0001)$.

\section{Effect on facial pain}

Blocked sinuses can cause facial pain, especially when pressure is applied on the corresponding facial area. The evolution of facial pain severity closely follows the patterns of headache evolution with only slight and not significant $(\mathrm{p}>0.05)$ reduction in the CP during the $1^{\text {st }}$ week of treatment followed by nearly $51 \%$ (SS, $\mathrm{p}<0.0001$ ) decrease between Days 7 and 14 compared to the initial mean value whereas in the TP group facial pain had subsided strongly and significantly on Day $3(-65 \%, p<0.0001)$ with further decrease on Day $6(-77 \%$, $\mathrm{p}<0.0001)$ and Day $14(-85 \%, \mathrm{p}<0.0001)$ compared to compared to baseline T0 mean value.

The difference in the evolution of pain intensity in TP group is equally highly significant when compared to CP with $-62 \%,-72 \%$ and $-70 \%$ severity difference on Day 3, 6, and 14 ( $\mathrm{p}<0.001$ ) (Figure 5 ).

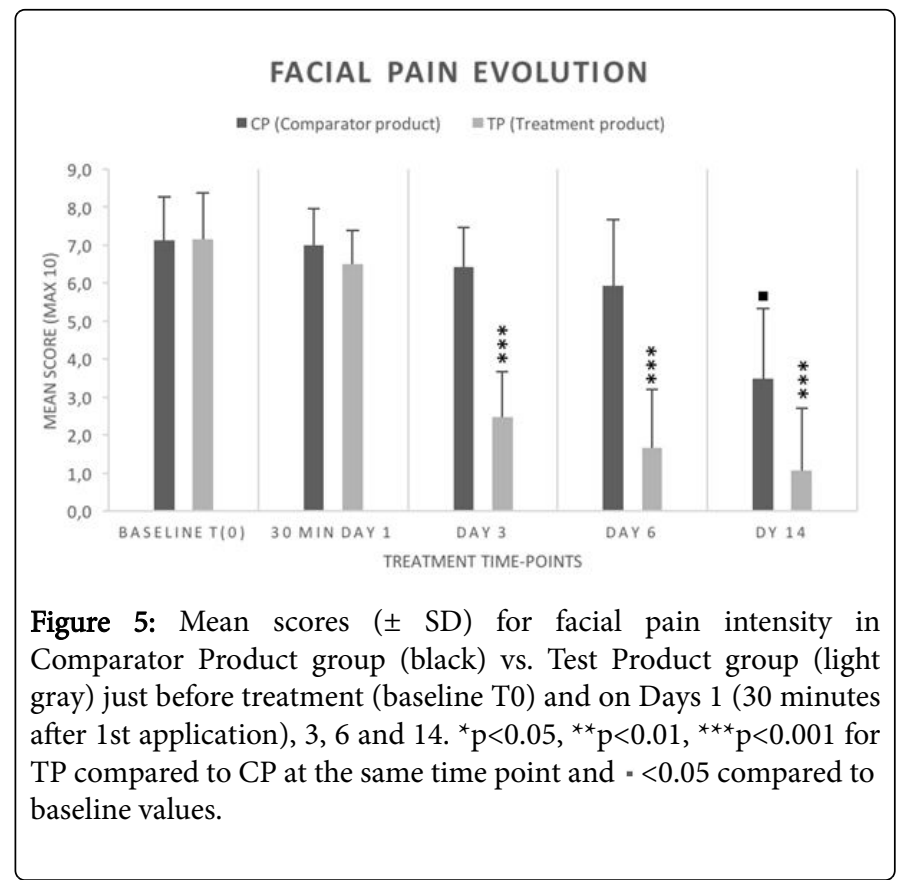

\section{Effect on the need for antibiotics}

For ethical reasons investigators were authorized to use antibiotics when they determined that patient condition was worsening and required antibiotherapy. The number and percentage of patients in each group and the duration of treatment are shown (Table 1).

\begin{tabular}{|c|c|c|}
\hline Parameter & $\begin{array}{ll}C P & \text { group } \\
(n=16) & \end{array}$ & $\begin{array}{ll}\text { TP } & \text { group } \\
(n=38) & \end{array}$ \\
\hline Number of patients treated & 3 & 1 \\
\hline Percentage $(\%)$ of patients & $18.75 \%$ & $2.63 \%$ \\
\hline Mean duration [within the study period] & 2.66 days & 2 days \\
\hline
\end{tabular}

Table 1: Need for and duration of antibiotic treatment in CP and TP groups.

These results show that the need for antibiotics was strongly reduced in the TP group (2.63\% patients) compared to the CP group (18.75\%). Antibiotherapy was initiated on the $6^{\text {th }}$ day of treatment for all patients who needed antibiotics. Mean duration of treatment was comparable in both groups (around 3 days).

\section{Changes in SNOT-22 parameters}

The Sino-Nasal Outcome Test (SNOT-22) is a validated patient reported measure of symptom severity and health-related Quality of Life in Sino-nasal conditions. The mean SNOT score of 22 parameters at the start of the study was 3.015 in the CP group compared to 3.042 in the TP group. At the end of the study, a strong reduction in SNOT score was observed in both groups (mean: 1.638 in CP and 1.105 in $\mathrm{TP}$ ) with a reduction of $45.67 \%$ in $\mathrm{CP}$ and $63.67 \%$ in $\mathrm{TP}$ group compared to the baseline values. The SNOT-22 results equally show that saline solution provides very good symptomatic relief from CRS.

\section{Product safety}

Safety was assessed by evaluating the number of patients reporting incidences of adverse events (AE) and/or serious adverse events (SAE) arising during or after the study and their assessment in respect to intensity, duration, pattern and causal relationship to the investigational product. Safety was further assessed by evaluating the patient compliance diary. The investigational products were both found to be totally safe as no treatment emergent adverse event (TEAE) was reported nor any cases of any adverse reaction were noticed during the study except for transient slight to moderate nasal irritation which is normal and due to the product's mode of action in some patients right after TP application. The test product was well tolerated and there were no notable changes in vital signs, physical examination and systemic examination. These results show that both investigational products (IPs) remain on the nasal surface; they are not absorbed and have no pharmacological, metabolic or immunological interactions with the underlying cells. Both products are therefore considered totally safe for topical application as a nasal spray for the treatment of rhinosinusitis.

\section{Complete recovery at the end of the study}

The percentage of patients scoring rhinorrhea, nasal congestion, headache and facial pain (key CRS symptoms recorded) as completely recovered (score $0 / 10$ ) or nearly recovered (score $1 / 10$ ) is shown below. This score was below $12.5 \%$ for all the parameters in the CP group. In the TP group, slight rhinorrhea was still present in $55.26 \%$ of the group population and nasal congestion in $39.47 \%$ of the group population at the end of the study while other symptoms had nearly disappeared. As rhinorrhea and congestion are easily tolerable symptoms and observed commonly in RS patients the efficacy of TP is considered superior to the CP (Table 2). 
Page 6 of 8

\begin{tabular}{|l|l|l|l|l|l|}
\hline Group & Score at end of study & Rhinorrhea & Nasal Congestion & Headache & Facial Pain \\
\hline CP $\%$ & $0 / 10$ & $0 \%$ & $6.25 \%$ & $12.50 \%$ & $12.50 \%$ \\
\hline TP $\%$ & $0 / 10$ & $15.79 \%$ & $23.68 \%$ & $89.47 \%$ & $39.47 \%$ \\
\hline CP $\%$ & 0 or $1 / 10$ & $6.25 \%$ & $12.50 \%$ & $12.50 \%$ & $12.50 \%$ \\
\hline TP $\%$ & 0 or $1 / 10$ & $44.74 \%$ & $60.53 \%$ & $97.74 \%$ & $84.21 \%$ \\
\hline
\end{tabular}

Table 2: \% of population in the CP $(n=16)$ and TP $(n=38)$ groups showing total recovery for individual RS symptoms at the end of the study; NB: we also assessed the number of patients scoring either 0 or 1 at the end of the study as $1 / 10$ may also reflect a drastic nearly complete recovery with only a hint of residual clinical sign.

\section{Product acceptability}

The results of product appreciation by patients and investigators are homogenous as none of the products was evaluated poor in efficacy. Treatment was evaluated by the patients and by the investigators as poor, faire, good, very good or excellent for each patient. None of the patients rated any treatment as poor. The efficacy was evaluated as fair by $21.88 \%$ patients in the $\mathrm{CP}$ compared to $6.58 \%$ in the $\mathrm{TP}$, as good by $50 \%$ in $\mathrm{CP}$ and $44.74 \%$ in $\mathrm{TP}$, as very good by $28.12 \%$ in CP compared to $32.89 \%$ in TP and as excellent by $0 \%$ in CP compared to $15.79 \%$ in TP. These results show that TP was much better accepted than CP for the treatment of RS.

\section{Discussion}

Chronic Rhinosinusitis (CRS) is a common health problem which significantly affects the sufferer's quality of life [12]. Rhinitis evolves towards sinusitis when the infection enters the nasal sinuses and becomes chronic when the infection persists beyond 12 weeks. Severe clinical signs appear when bacteria colonize the sinuses as those are extremely difficult to dislodge $[13,14]$. Unfortunately, all current and even emerging treatment options are intended to ease one or several clinical symptoms often in association with other therapies including saline nasal washes, topical or oral antibiotics, nasal decongestants, steroids and anti-inflammatory drugs [13]. New emerging options include antimycotics, anti-IgE, anti-IL5, new antihistamines, complementary and alternative medicine, immunosuppressant medications, leukotriene inhibitors, phytotherapy, probiotics and proton pump inhibitors [15-22]. Despite such abundance of treatments available one wonders why none of them is really working and why even today, we don't have a cure for CRS. In theory it would be sufficient to clean the sinuses to allow their contents to drain and to remove all the contaminants from the nasal cavity to allow natural healing. Recent findings clearly prove that bacterial biofilm which obstructs sinus openings in as many as 75\% CRS patients is the main cause of persistent CRS [6,23]. A biofilm is a three-dimensionally structured specialized community of adherent microorganisms surrounded by an extracellular polymeric substance (EPS), a strong bodyguard for hidden microorganisms. Biofilms contain multiple bacterial and/or fungal species in a single community and survive symbiotically with protective and metabolic collaboration between different species of microorganisms [24]. During the initial stage of the condition (2-4 weeks) when the biofilm is not yet too robust, it may probably be dispersed with regular and frequent saline washes or with sea water containing $3.2-3.4 \% \mathrm{NaCl}$ (upper threshold cytotoxic concentration limits) but once it becomes well adhered to the mucus membrane and stronger with time, it is highly resistant to any topical or systemic treatments and should ideally be removed mechanically. Saline solution used as nasal wash with large volume-low pressure delivery devices or as multiple irrigations is still considered one of the best and safest treatments to ease CRS symptoms as these treatments are safe and have a positive benefit/risk ratio $[25,26]$. The mode of action, beyond the flushing mechanical action of saline solutions is not clearly established but regular nasal washes may help clean nasal passage, improve respiration, ciliary beats or reduce microbial load thereby alleviating CRS symptoms [27].

In this study, when physiological saline was used as a regular spray without interruption during 14 days, it provided good symptomatic relief, minimizing rhinorrhea, nasal congestion, headache, facial pain and a remarkable reduction of sinusitis severity assessing SNOT-22 parameters but the improvement was limited to maximum $50 \%$ for almost all parameters in the CP group. This is comprehensible as saline solution has no effect on biofilm. Normally, disruption of biofilm should produce instant and strong nasal discharge, indicative of ruptured biofilm and sinus opening and drainage but this phenomenon was not observed in the $\mathrm{CP}$ group as normal saline has no osmotic but only cleaning properties and sea water containing $3.2-3.4 \% \mathrm{NaCl}$ is not sufficiently osmotic to exert osmotic pressure that would lead to disruption of biofilms. The salt concentration cannot be increased above $3.4 \%$, as the solution then becomes too irritable to the nasal mucosa and may cause severe inflammation and nasal mucosa damage. A few solutions containing higher salt concentrations such as pure or diluted sea water exist on the market as symptomatic treatments only [27].

The physiopathology of CRS demands for a treatment to be effective that it exert a positive osmotic pressure over sinus-blocking biofilm membranes to open the sinuses and to drain the sinus contents. Almost all chemicals are either irritant or cytotoxic for the nasal mucosa and therefore cannot be used on the nasal surface. Similarly, any systemic drug will have difficulties to achieve therapeutic concentration inside the sinuses without side effects. The results of this study show that topical application of filmogen FPG strongly increases rhinorrhea in the TP group especially 30 minutes after the $1^{\text {st }}$ product application. Nasal discharge then remained consequential up to Day 3 followed by progressive reduction till the end of the treatment. If this overabundant but time-limited excess outflow was only due to the osmotic properties of the TP, it would have continued throughout the entire period of treatment. Therefore, it can be reasonably concluded that TP forms a strongly osmotic film over the sinus openings, generating a positive osmotic pressure to draw hypotonic intra-sinusal liquid, thereby opening the sinuses and draining sinus contents, which is manifested as strong initial rhinorrhea. This sinus drainage continues as long as the sinuses are not totally cleared. Relieving intra- 
sinus pressure reduces nasal congestion, headache, facial pain upon pressure and all SNOT parameters and in consequence leads to reduced antibiotic needs and improved quality of life for the patients. As antibiotics are generally prescribed when investigators observed aggravation associated with probable complication by secondary bacterial infection, a minimized requirement for antibiotics in the TP group indicates powerful antimicrobial properties exerted by the TP. It is postulated that cleaning the nasal mucosa surface of microbial and other contaminants accelerates natural healing process and prevented bacterial infection.

All currently used topical drugs against CRS such as antiseptics, antibiotics, anti-inflammatory drugs, cytokine inhibitors or hypertonic saline solutions cannot reach the sinuses often remain on the nasal surface and provide only temporary symptomatic relief and the benefit to harm ratio of these drugs is still debatable $[28,20]$.

A few patients of this study complained about moderate nasal irritation right after each TP application, lasting for about 15-30 seconds. This may be attributable to the fact that FPG solution is osmotic and draws intracellular hypotonic liquid as soon as it is sprayed as a film over the nasal mucosa. This may lead to very brief cellular dehydration and irritation. VB-Gy concentration in FPG was thus adjusted to the limit where it conserves sufficient filmogen and osmotic properties without generating prolonged cellular dehydration which may cause severe nasal irritation and eventual cellular death or nasal lesions.

To prevent instant dilution of the film a small quantity of VB-Gy binding plant extracts rich in polymeric tannins was added to it as experiments and studies conducted by our laboratory have shown that certain specific tannins bind to the VB-Gy molecules and create a flexible film with increased resistance to dilution resulting in improved duration of action [29]. Tannins are large, inert and highly branched molecules which do not interact with the cellular structures are noncytotoxic even at higher concentrations and they are easily expelled with hypotonic liquid flow once the FPG film is disintegrated. The plant extracts or their associations were selected based on their capacity to bind to VB-Gy and due to their highly-branched structure only very small quantities are required to obtain a flexible FPG film. The concentrations of plant extracts used in the final preparation do not have any pharmacological effect as these concentrations are considerably lower than the minimum concentrations or doses recommended to obtain pharmacological effects [30]. In this study, the FPG product was applied topically on the nasal mucosa as a filmogen spray and in the absence of any nasal mucosa damage or adverse effects, it is concluded that the TP acts solely topically on the nasal mucosa surface without altering any cellular parameters. Although, the incorporated polymers bind with VB-Gy molecules, topical polymer binding with free floating proteins such as viral glycoproteins, bacterial fibronectin, cytokines or cell membrane proteins on the nasal surface cannot be excluded. Such filmogen polymer containing, osmotic, cellfriendly and non-irritant solutions can also be used as a liquid bandage to treat any live damaged biological surface [3,31].

It should be noted that cleaning the nasal sinuses is the prerequisite to treat CRS yet up till now there was no mechanical, chemical or physiological treatment capable of providing nearly instant relief although several efforts have been made (application of $\mathrm{N}$ acetylcysteine, antibiotics, steroids and sea water saline with betamethasone) but without success [23,32-36]. Therefore, the discovery of FPG, a new generation of mechanically acting filmogen, cell-friendly, non-irritant, topically applicable solution devoid of side effects which can be used as an osmotic bandage on the nasal mucosa should be considered as a major breakthrough for the treatment of CRS.

\section{Acknowledgments}

This clinical study was commissioned and funded by the Naturveda Laboratory.

\section{References}

1. Halawi AM, Smith SS, Chandra RK (2013) Chronic rhinosinusitis: Epidemiology and cost. Allergy Asthma Proc 34: 328-334.

2. Dlugaszewska J, Leszczynska M, Lenkowski M, Tatarska A, Pastusiak T, et al. (2016) The pathophysiological role of bacterial biofilms in chronic sinusitis. Eur Arch Otorhinolaryngol 273: 1989-1994.

3. Ferguson BJ, Stolz DB (2005) Demonstration of biofilm in human bacterial chronic rhinosinusitis. Am J Rhinol 19: 452-457.

4. Tan BK, Kern RC, Schleimer RP, Schwartz BS (2013) Chronic rhinosinusitis: The unrecognized epidemic. Am J Respir Crit Care Med 188: 1275-1277.

5. Tomassen P, Van Zele T, Zhang N, Perez-Novo C, Van Bruaene N, et al. (2011) Pathophysiology of chronic rhinosinusitis. Proc Am Thorac Soc 8: 115-120.

6. Lam K, Schleimer R, Kern RC (2015) The etiology and pathogenesis of Chronic Rhinosinusitis: A review of current hypotheses. Curr Allergy Asthma Rep 15: 41.

7. Suh JD, Kennedy DW (2011) Treatment options for chronic rhinosinusitis. Proc Am Thorac soc 8: 132-140.

8. Liang J, Lane AP (2013) Topical drug delivery for chronic rhinosinusitis. Curr Otorhinolaryngol Rep 1: 51-60.

9. Shrivastava R (2000) Non-solid composition for local application.

10. Shrivastava R, Shrivastava L (2016) Composition for topical application comprising glycerol and tannins.

11. Browne JP, Hopkins C, Slack R, Cano SJ (2007) The sino-nasal outcome test (snot): Can we make it more clinically meaningful. Otolaryngol Head Neck Surg 136: 736-741.

12. Chen H, Katz PP, Shiboski S, Blanc PD (2005) Evaluating change in health-related quality of life in adult rhinitis: Responsiveness of the rhinosinusitis disability index. Health Qual Life Outcomes 3: 68.

13. Statham MM, Seiden A (2005) Potential new avenues of treatment for chronic rhinosinusitis: An anti-inflammatory approach. Otolaryngol Clin North Am 38: 1351-1365.

14. Piromchai P, Kasemsiri P, Laohasiriwong S, Thanaviratananich S (2013) Chronic rhinosinusitis and emerging treatment options. Int J Gen Med 6: 453-464.

15. Charakorn N, Snidvongs K (2016) Chronic sphenoid rhinosinusitis: Management challenge. J Asthma Allergy 9: 199-205.

16. Li N, Peters AT (2015) Chronic rhinosinusitis management beyond intranasal steroids and saline solution irrigations. Allergy Asthma Proc 36: 339-343.

17. Zhang YL, Han DH, Kim DY, Lee CH, Rhee CS (2017) Role of interleukin-17A on the chemotactic responses to CCL7 in a murine allergic rhinitis model. PLoS One 12: e0169353.

18. Bachert C, Holtappels G (2015) Pathophysiology of chronic rhinosinusitis, pharmaceutical therapy options. GMS Curr Top Otorhinolaryngol Head Neck Surg 14: Doc09.

19. Yavuz ST, Koc O, Gok AF, Hawley J, O’Brien C, et al. (2016) Proceedings of 4 th pediatric allergy and asthma meeting (PAAM). Clin Transl Allergy 6: $1-60$.

20. Kennedy JL, Borish L (2013) Chronic rhinosinusitis and antibiotics: The good, the bad, and the ugly. Am J Rhinol Allergy 27:467-472.

21. Passali D, Cambi J, Passali FM, Bellussi LM (2015) Phytoneering: A new therapy for rhinosinusitis. Acta Otorhinolaryngol Ital. 35: 1-8. 
Citation: Shrivastava RM, Tourret E, Schutte H, Shrivastava RS and Roura F (2017) Clinical Efficacy of a New Filmogen Polymeric Glycerol Solution for the Treatment of Rhinosinusitis. J Clin Respir Dis Care 3: 130. doi:10.4172/2472-1247.1000130

Page 8 of 8

22. Ghazi-Moghadam K, Inançlı HM, Bazazy N, Plinkert PK, Efferth T, et al. (2012) Phytomedicine in otorhinolaryngology and pulmonology: Clinical trials with herbal remedies. Pharmaceuticals (Basel) 5: 853-874.

23. Tatar EC, Tatar I, Ocal B, Korkmaz H, Saylam G, et al. (2012) Prevalence of biofilms and their response to medical treatment in chronic rhinosinusitis without polyps. Otolaryngol Head Neck Surg 146: 669-675.

24. Wolcott R, Costerton JW, Raoult D, Cutler SJ (2013) The polymicrobial nature of biofilm infection. Clin Microbiol Infect 19: 107-112.

25. Rachel BC, Devyani L (2013) Update on the management of chronic rhinosinusitis. Infect Drug Resist 6: 1-14

26. Schmid A, Salathe M (2011) Ciliary beat co-ordination by calcium. Biol Cell 103: 159-169.

27. Bonnomet A, Luczka E, Coraux C, de Gabory L (2016) Non-diluted seawater enhances nasal ciliary beat frequency and wound repair speed compared to diluted seawater and normal saline. Int Forum Allergy Rhinol 6: 1062-1068.

28. Watelet JB, Eloy PH, Van Cauwenberge PB (2007) Drug management in chronic rhinosinusitis: Identification of the needs. Ther Clin Risk Manag 3: $47-57$.

29. Shrivastava R, Cucuat N, Rousse M, Weigand T, Neto P, et al. (2014) A new generation of topical chronic wound treatments containing specific MMP inhibitors. Chro Wound Car Manag Rese1: 31-40.

30. Regulation (EC) No 1924/2006 of the European Parliament and of the Council of 20 December 2006 on nutrition and health claims made on foods.
31. Shrivastava R (2011) A pilot clinical trial to evaluate the efficacy of a topical antiviral osmotically active hypertonic solution for the treatment of influenza virus induced sore throat. J Clinic Trials 1: 1-7.

32. Blasi F, Page C, Rossolini GM, Pallecchi L, Matera MG, et al. (2016) The effect of N-acetylcysteine on biofilms: Implications for the treatment of respiratory tract infections. Respir Med 117: 190-7.

33. Head K, Chong LY, Piromchai P, Hopkins C, Philpott, et al. (2016) Systemic and topical antibiotics for chronic rhinosinusitis. Coch Data Syst Rev 26: CD011994.

34. Cirkovic I, Pavlovic B, Bozic DD, Jotic A, Bakic L, et al. (2017) Antibiofilm effects of topical corticosteroids and intranasal saline in patients with chronic rhinosinusitis with nasal polyps depend on bacterial species and their biofilm-forming capacity. Eur Arch Otorhinolaryngol. 4: 1897-1903.

35. Chong LY, Head K, Hopkins C, Philpott C, Glew S, et al. (2016) Saline irrigation for chronic rhinosinusitis. Coch Data Syst Rev 4: CD011995.

36. Ong KY, Lim WC, Ooi SM, Loh ZH, Kong MC, et al. (2017) Stability of a novel corticosteroid nasal irrigation solution: Betamethasone 17-valerate added to extemporaneously prepared nasal irrigation solutions. Int Forum Allergy Rhinol 7: 494-501. 